\title{
A Simple Route to Benz[a]acridinium Salts
}

\section{Horst Hartmann}

Department Chemie, Technische Universität Dresden, 01062 Dresden, Germany

Reprint requests to Prof. Dr. Horst Hartmann. Fax: ++49-351-4633 9485. E-mail: Hartmann@ iapp.de

Z. Naturforsch. 2011, 66b, 711-714; received May 5, 2011

Dedicated to Prof. Dr. Dr. hc mult. Siegfried Hünig to the occasion of his $90^{\text {th }}$ birthday

By reaction of $N$-substituted $N$-phenyl-2-naphthylamimes with the Vilsmeier reagent $N$-substituted benz[a]acridinium salts are formed in satisfactory yields.

Key words: Vilsmeier Reaction, $N$-Substituted 2-( $N$-Phenyl)-naphthylamines, Benz[a]acridinium Perchlorates

\section{Introduction}

As has been known for a long time, the Vilsmeier reaction (VR), usually performed with a mixture of DMF and $\mathrm{POCl}_{3}$, is a versatile method for preparing aromatic or heteroaromatic aldehydes in an enormous variety [1]. For instance, by starting with 4unsubstituted $N, N$-dialkylanilines 1a, $N, N$-disubstituted 4-aminobenzaldehydes 2 or, under more rigorous conditions, corresponding $\mathrm{N}, \mathrm{N}$-disubstituted 4amino-terephthalaldehydes $\mathbf{3}$ are formed [2]. However, by starting with 4 -substituted $N, N$-dialkylanines $\mathbf{1 b}$, instead of expected $N, N$-disubstituted 2-amino-benz-

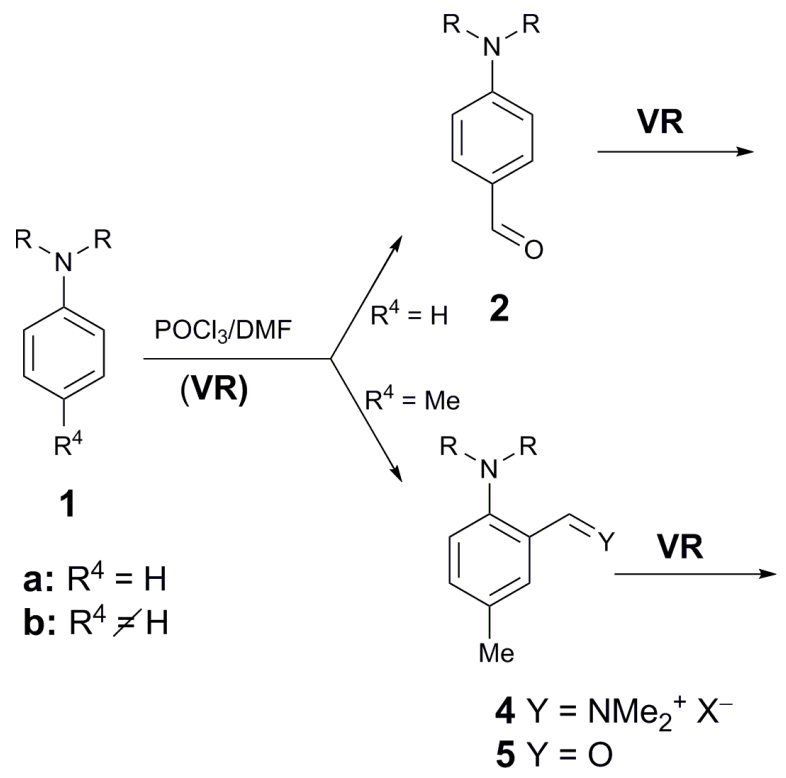

aldehydes $\mathbf{5}$, the 1,3,3,4-tetrahydro-3-quinazolinium salts 6 were obtained [3]. These compounds arise by cyclisation of the corresponding dimethyliminium salts 4 intermediately formed in the course of the VR and can be regarded as undesired by-products in the Vilsmeier aldehyde synthesis (Scheme 1). In contrast, the mono-formyl compounds $\mathbf{2}$ and the bis-formyl derivatives $\mathbf{3}$ are important starting materials for a variety of products with some practical interest, such as organic dyes with intense absorption in the visible or near infrared region [4], with highly non-linear optical properties [5], or with a high two-photon cross section [6].<smiles>[R]N([R])c1ccc(C=O)cc1C=O</smiles>

$$
3
$$<smiles>[X]c1ccc2c(c1)C[N+]([R16])([R4])CN2[R]</smiles>

6

Scheme 1. VR = Vilsmeier reaction. 


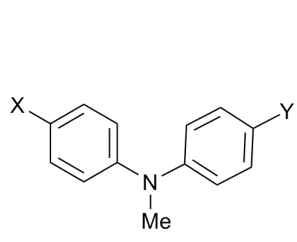

7

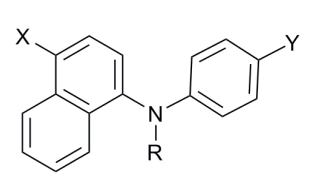

$9 \mathrm{R}=\mathrm{Me}$

$10 \mathrm{R}=\mathrm{Ph}$

Scheme 2 .

The Vilsmeier formylation of $N, N$-dialkylanilines $\mathbf{1}$ outlined here starts similarly with $N$-monoaryl- and $N, N$-diaryl-substituted aniline derivatives and gives rise to the formation of corresponding $N$-monoaryl- and $N, N$-diaryl-substituted aldehydes or their dimethyliminium salt precursors (Scheme 2). Thus, $\mathrm{N}$-methyl-diphenylamine 7a, triphenylamine $\mathbf{8 a}, \mathrm{N}$ methyl- $N$-phenyl-1-naphthylamine 9a, and $N, N$-diphenyl-1-naphthylamine 10a were converted by a VR into the corresponding $N$-methyl- $N$-phenyl-4-aminobenzaldehyde 7b [7], $N, N$-diphenyl-4-aminobenzaldehyde 8b [8], $N$-methyl- $N$-phenyl-4-amino-1-naphthaldehyde 9b [9], and $N, N$-diphenyl-4-amino-1-naphthaldehyde 10b [10], respectively. Under more rigorous conditions the bis-formyl derivatives $\mathbf{7 c - 1 0 c}$ as well as the tris-formyl derivative $\mathbf{8 d}$ were formed [8a, 11].

\section{Results and Discussion}

A quite different result has been found, however, when $N$-phenyl-substituted 2-naphthylamines 12 were used as reactants in the VR. Instead of the corresponding formyl compounds $\mathbf{1 4}$ (or their corresponding dimethyliminium salt precursors 13) benz[a]acridinium salts of the general structure $\mathbf{1 5}$ were obtained (Scheme 3).

The reaction can be advantageously performed by heating a mixture of the Vilsmeier reagent with the appropriate $N$-phenyl-substituted 2-naphthylamine $\mathbf{1 2}$ at $80-90{ }^{\circ} \mathrm{C}$ for several hours and quenching the reaction mixture after cooling by pouring into methanol. The benz[a]acridinium salts $\mathbf{1 5}$ so formed could be isolated after addition of aqueous perchloric acid to the reaction mixture and were obtained in rather high yields.

The necessary $N$-substituted $N$-phenyl-2-naphthylamines $\mathbf{1 2}$ are commercially not available, but they can be synthesised easily from the $N$-unsubstituted $N$ phenyl-2-naphthylamine parent compound 11 by addition of an appropriate alkylating agent of the general formula R-X before starting the Vilsmeier formylation. The $N, N$-diphenyl-2-naphthylamine $10 \mathrm{~g}$ was prepared, however, by a palladium-catalysed $C-N$ cross coupling from $N$-phenyl-2-naphthylamine and bromobenzene [12].

The benz[a]acridinium salts $\mathbf{1 5}$ so prepared are paleyellow solids. Their structures follow unambiguously from the analytic data. For instance, all salts exhibit in the mass spectra $\mathrm{m} / \mathrm{z}$ values which are in accord with the cationic moieties, and in the ${ }^{1} \mathrm{H}$ NMR spectra they show characteristic signals between $\delta=7.8-9.5$ and $\sim 10.0 \mathrm{ppm}$. Whereas the former signals originate from the protons at the carbocyclic rings, the latter signals can be attributed to the protons at $\mathrm{C} 12$ of the heterocyclic azine ring in the benzo[c]acridinium salts $\mathbf{1 5}$.

In contrast to acridines [13], acridinium salts are hitherto sparsely known and usually prepared by alkylation of their non-ionic parent compounds [14]. Because they are of some interest for various applications, e.g. as chemotherapeutics (e.g. Acriflavine), as fluorescent stains [15], or as organic dyes [16], the simple access described will make further studies on these compounds much easier.
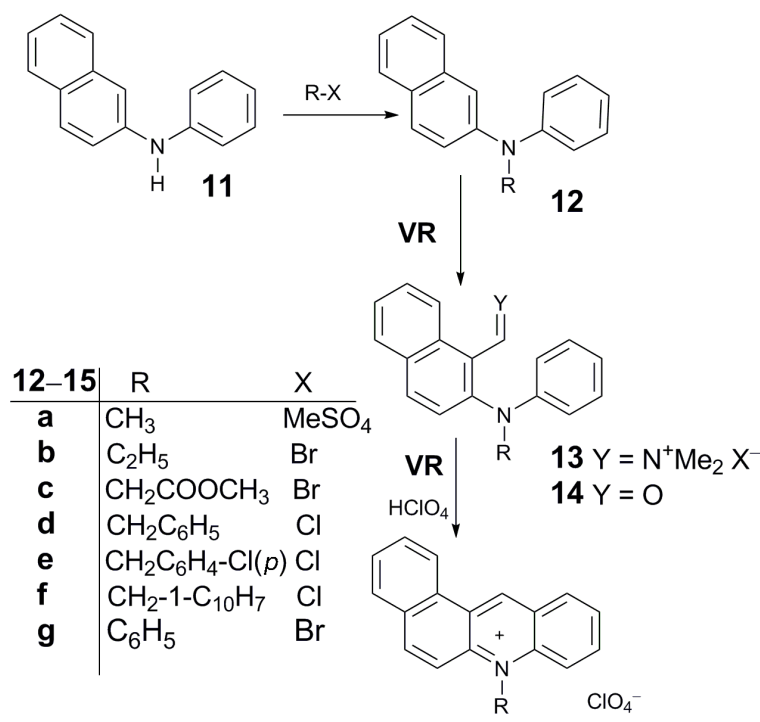

Scheme 3.
15 


\section{Experimental Section}

${ }^{1} \mathrm{H}$ NMR spectra were recorded in trifluoroacetic acid with a Bruker DRX $500 \mathrm{P}$ instrument at $500.13 \mathrm{MHz}$. The melting points were measured with a Boetius heatingtable microscope. Mass spectra were recorded with a Bruker Esquire-LC 00084 instrument at $10 \mathrm{~V}$ with electrospray ionisation using methanol containing $0.1 \%$ ammonium acetate.

\section{Preparation of N,N-diphenyl-2-naphthylamine (12g)}

To a solution of $N$-phenyl-2-naphthylamine $(\mathbf{1 1}, 54.8 \mathrm{~g}$, $0.25 \mathrm{~mol})$, bromobenzene $(39.3 \mathrm{~g}, 0.25 \mathrm{~mol})$ and Na-tert.butanolate $(28.8 \mathrm{~g}, 0.3 \mathrm{~mol})$ in toluene $(500 \mathrm{~mL})$ a solution of palladium-bis-(tri-tert.-butylphosphane) $(10 \mathrm{~mL}, 10 \%)$ in toluene was added dropwise under argon at r.t. After refluxing the resulting mixture for $8 \mathrm{~h}$, it was cooled to r.t. and mixed with water $(500 \mathrm{~mL})$. The organic phase was separated and the aqueous solution extracted twice with diethyl ether $(250 \mathrm{~mL})$. The organic solutions were combined, dried with $\mathrm{MgSO}_{4}$, and evaporated in vacuo. The product so obtained in a yield of $74 \mathrm{~g}$ was purified by recrystallisation from a toluene/cyclohexane mixture. M. p. $120{ }^{\circ} \mathrm{C}$ (Lit. [17]: 120 $\left.121{ }^{\circ} \mathrm{C}\right) .-{ }^{1} \mathrm{H}$ NMR $\left(400 \mathrm{MHz}, \mathrm{CDCl}_{3}\right): \delta=7.05(\mathrm{dt}, 2 \mathrm{H})$, $7.12(\mathrm{t}, 1 \mathrm{H}), 7.15(\mathrm{t}, 2 \mathrm{H}), 7.25-7.27(\mathrm{~m}, 5 \mathrm{H}), 7.30(\mathrm{t}, 1 \mathrm{H})$, $7.37(\mathrm{dt}, 2 \mathrm{H}), 7.42(\mathrm{t}, 1 \mathrm{H}), 7.58(\mathrm{~d}, J=10.0 \mathrm{~Hz}, 1 \mathrm{H}), 7.73$ (dd, $J=11.2, \mathrm{~J}=4 \mathrm{~Hz}, 2 \mathrm{H}$ ).

\section{Preparation of the benz[a]acridinium perchlorates 15 (General procedure)}

To a solution of $\mathrm{N}$-phenyl-2-naphthylamine $(\mathbf{1 1}, 2.2 \mathrm{~g}$, $10 \mathrm{mmol})$ in DMF $(25 \mathrm{~mL})$ the corresponding alkylating reagent $\mathrm{R}-\mathrm{X}(15 \mathrm{mmol})$ was added and the resulting mixture heated at $120{ }^{\circ} \mathrm{C}$ for $30 \mathrm{~min}$. After cooling, $\mathrm{POCl}_{3}(3.2 \mathrm{~g}$, $20 \mathrm{mmol}$ ) was added dropwise to the reaction mixture which was subsequently heated at $90{ }^{\circ} \mathrm{C}$ for $2 \mathrm{~h}$ and then cooled to r.t. After addition of methanol $(50 \mathrm{~mL})$, perchloric acid $(70 \%, 2 \mathrm{~mL})$ was added to the resulting mixture under cooling. The precipitate formed after addition of some ethyl acetate was isolated by suction and recrystallised, after drying at air, from acetic acid. The following benz[a]acridinium perchlorates were prepared:

7-Methyl-benzo[a]acridinium perchlorate $(\mathbf{1 5 a})$ in a yield of $80 \%$. M.p. $320-324{ }^{\circ} \mathrm{C}$. $-{ }^{1} \mathrm{H}$ NMR $(400 \mathrm{MHz}$, $\left.\mathrm{CF}_{3} \mathrm{COOH}\right): \delta=5.32\left(\mathrm{~s}, 3 \mathrm{H}, \mathrm{CH}_{3}\right), 8.35(\mathrm{t}, 1 \mathrm{H}), 8.46(\mathrm{t}$, $2 \mathrm{H}), 8.56(\mathrm{~d}, J=10.0 \mathrm{~Hz}, 1 \mathrm{H}), 8.70-8.81(\mathrm{~m}, 2 \mathrm{H}), 8.95$ $(\mathrm{dd}, J=12.8 \mathrm{~Hz}, J=4.0 \mathrm{~Hz}, 2 \mathrm{H}), 9.08(\mathrm{~d}, J=12.8 \mathrm{~Hz}, 1 \mathrm{H})$, $9.39(\mathrm{~d}, J=10.8 \mathrm{~Hz}, 1 \mathrm{H}), 10.76(\mathrm{~s}, 1 \mathrm{H}) .-\mathrm{MS}((+) \mathrm{ESI})$ : $m / z=244.0$ (calcd. 244.31 for $\mathrm{C}_{18} \mathrm{H}_{14} \mathrm{~N}^{+},\left[\mathrm{M}-\mathrm{ClO}_{4}^{-}\right]^{+}$). $\mathrm{C}_{18} \mathrm{H}_{14} \mathrm{ClNO}_{4}$ (343.76): calcd. C 62.89, $\mathrm{H} 4.10, \mathrm{Cl} 10.31$, N 4.20, O 18.62; found C 62.44, H 4.18, N 4.20.

7-Ethyl-benzo[a]acridinium perchlorate $(\mathbf{1 5 b})$ in a yield of $86 \%$. M. p. $293{ }^{\circ} \mathrm{C}$. $-{ }^{1} \mathrm{H}$ NMR (400 MHz, $\left.\mathrm{CF}_{3} \mathrm{COOH}\right)$ : $\delta=1.80\left(\mathrm{t}, 3 \mathrm{H}, \mathrm{CH}_{3}\right), 5.99\left(\mathrm{q}, 2 \mathrm{H}, \mathrm{CH}_{2}\right), 7.78(\mathrm{t}, 1 \mathrm{H}), 7.81-$ $7.93(\mathrm{~m}, 2 \mathrm{H}), 8.00(\mathrm{~d}, J=7.9 \mathrm{~Hz}, 1 \mathrm{H}), 8.15(\mathrm{~d}, J=9.7 \mathrm{~Hz}$, $1 \mathrm{H}), 8.26(\mathrm{t}, 1 \mathrm{H}), 8.37(\mathrm{~d}, J=9.2 \mathrm{~Hz}, 1 \mathrm{H}), 8.44(\mathrm{~d}, J=8.2 \mathrm{~Hz}$, $1 \mathrm{H}), 8.53(\mathrm{~d}, J=9.8 \mathrm{~Hz}, 1 \mathrm{H}), 8.84(\mathrm{~d}, J=8.3 \mathrm{~Hz}, 1 \mathrm{H})$, 10.19 (s, 1H). - MS ((+)ESI): $m / z=258.1$ (calcd. 258.34; for $\left.\mathrm{C}_{19} \mathrm{H}_{16} \mathrm{~N}^{+},\left[\mathrm{M}-\mathrm{ClO}_{4}^{-}\right]^{+}\right),-\mathrm{C}_{19} \mathrm{H}_{19} \mathrm{ClNO}_{4}$ (357.79): calcd. C 63.78, H 4.51, Cl 9.91, N 3.91, O 17.89; found C 63.64, $\mathrm{H} 4.81, \mathrm{~N} 4.55$.

7-Methoxycarbonylmethyl-benzo[a]acridinium perchlorate $(15 c)$ in a yield of $75 \%$. M. p. $290-292{ }^{\circ} \mathrm{C} .-{ }^{1} \mathrm{H}$ NMR (400 MHz, $\left.\mathrm{CF}_{3} \mathrm{COOH}\right): \delta=4.47\left(\mathrm{~s}, 3 \mathrm{H}, \mathrm{CH}_{3}\right), 6.71(\mathrm{~s}, 2 \mathrm{H}$, $\left.\mathrm{H}_{2}\right), 8.39(\mathrm{t}, 1 \mathrm{H}), 8.49-8.61(\mathrm{~m}, 4 \mathrm{H}), 8.74-8.85(\mathrm{~m}, 2 \mathrm{H})$, $9.03(\mathrm{~d}, J=11.2 \mathrm{~Hz}, 1 \mathrm{H}), 9.12(\mathrm{~d}, J=12.8 \mathrm{~Hz}, 1 \mathrm{H}), 9.43$ (d, $J=11.2 \mathrm{~Hz}, 1 \mathrm{H}), 10.87(\mathrm{~s}, 1 \mathrm{H}) .-\mathrm{MS}((+) \mathrm{ESI}): m / z=$ 302.1 (calcd. 302.35 for $\mathrm{C}_{20} \mathrm{H}_{16} \mathrm{NO}_{2}^{+},\left[\mathrm{M}-\mathrm{ClO}_{4}^{-}\right]^{+}$). $\mathrm{C}_{20} \mathrm{H}_{16} \mathrm{ClNO}_{6}$ (401.80): calcd. C 59.78, H 4.01, Cl 8.82, N 3.49, O 23.89; found C 59.24, H 4.01, N 3.45.

7-Benzyl-benzo[a]acridinium perchlorate $(\mathbf{1 5 d})$ in a yield of $92 \%$. M.p. $288-289{ }^{\circ} \mathrm{C}$. $-{ }^{1} \mathrm{H} \mathrm{NMR}(400 \mathrm{MHz}$, $\left.\mathrm{CF}_{3} \mathrm{COOH}\right): \delta=7.02\left(\mathrm{~s}, 2 \mathrm{H}, \mathrm{CH}_{2}\right), 7.56(\mathrm{~d}, J=12.0 \mathrm{~Hz}$, $2 \mathrm{H}), 7.80(\mathrm{~m}, 2 \mathrm{H}), 8.36(\mathrm{t}, 1 \mathrm{H}), 8.47-8.61(\mathrm{~m}, 3 \mathrm{H}), 8.72$ $(\mathrm{t}, 1 \mathrm{H}), 8.20(\mathrm{~d}, J=12.0 \mathrm{~Hz}, 1 \mathrm{H}), 8.99(\mathrm{t}, 2 \mathrm{H}), 9.46$ $(\mathrm{d}, J=11.6 \mathrm{~Hz}, 1 \mathrm{H}), 10.88(\mathrm{~s}, 1 \mathrm{H}) .-\mathrm{MS}((+) \mathrm{ESI})$ : $m / z=320.2$ (calcd. 320.41 for $\mathrm{C}_{24} \mathrm{H}_{18} \mathrm{~N}^{+},\left[\mathrm{M}-\mathrm{ClO}_{4}^{-}\right]^{+}$). $\mathrm{C}_{24} \mathrm{H}_{18} \mathrm{ClNO}_{4}$ (419.86): calcd. C 68.66, $\mathrm{H} \mathrm{4.32,} \mathrm{Cl} 8.44$, N 3.34; O 15.24; found C 69.04, H 4.31, N 3.54.

7-(4-Chlorobenzyl)-benzo[a]acridiniumperchlorate (15e) in a yield of $96 \%$. M.p. $299-300{ }^{\circ} \mathrm{C} .-{ }^{1} \mathrm{H}$ NMR $(400 \mathrm{MHz}$, $\left.\mathrm{CF}_{3} \mathrm{COOH}\right): \delta=6.62\left(\mathrm{~s}, 2 \mathrm{H}, \mathrm{CH}_{2}\right), 7.13(\mathrm{~d}, J=11.2 \mathrm{~Hz}$, $2 \mathrm{H}), 7.42(\mathrm{~d}, J=11.2 \mathrm{~Hz}, 2 \mathrm{H}), 8.01(\mathrm{t}, 1 \mathrm{H}), 8.11-8.22(\mathrm{~m}$, 4H), $8.36(\mathrm{~m}, 2 \mathrm{H}), 8.67(\mathrm{t}, 2 \mathrm{H}), 9.09(\mathrm{~d}, J=10.8 \mathrm{~Hz}, 1 \mathrm{H})$, 10.49 (s, 1H). - MS ((+)ESI): $m / z=354.2,355.1,356.1$, 357.1 (calcd. 354.85 for $\mathrm{C}_{24} \mathrm{H}_{17} \mathrm{ClN}^{+},\left[\mathrm{M}-\mathrm{ClO}_{4}^{-}\right]^{+}$). $\mathrm{C}_{24} \mathrm{H}_{17} \mathrm{Cl}_{2} \mathrm{NO}_{4}$ (454.30): calcd. C 63.45, $\mathrm{H} 3.77, \mathrm{Cl} 15.61$, N 3.08, O 14.09; found C 63.49, H 3.31, N 3.20.

7-(1-Naphthylmethyl)-benz[a]acridinium perchlorate (15f) in a yield of $90 \%$. M.p. $297-300{ }^{\circ} \mathrm{C} .-{ }^{1} \mathrm{H}$ NMR (400 MHz, $\left.\mathrm{CF}_{3} \mathrm{COOH}\right): \delta=7.07\left(\mathrm{~s}, 3 \mathrm{H}, \mathrm{CH}_{2}\right), 7.79(\mathrm{t}, 1 \mathrm{H})$, 7.90 (t, 1H), $7.97-8.20(\mathrm{M}, 7 \mathrm{H}), 8.29$ (d, $J=4.8 \mathrm{~Hz}, 2 \mathrm{H})$, $8.35(\mathrm{~d}, J=10.8 \mathrm{~Hz}, 1 \mathrm{H}), 8.57$ (d, $J=12.8 \mathrm{~Hz}, 1 \mathrm{H}), 8.73$ (d, $J=11.2 \mathrm{~Hz}, 1 \mathrm{H}), 9.15, \mathrm{~d}, J=11.6 \mathrm{~Hz}, 1 \mathrm{H}), 10.57(\mathrm{~s}, 1 \mathrm{H})$. MS ((+)ESI): $\mathrm{m} / \mathrm{z}=370.2$ (calcd. 370.47 for $\mathrm{C}_{28} \mathrm{H}_{20} \mathrm{~N}^{+}$, [M$\left.\left.\mathrm{ClO}_{4}^{-}\right]^{+}\right) .-\mathrm{C}_{18} \mathrm{H}_{14} \mathrm{ClNO}_{4}$ (469.92): calcd. C 71.57, $\mathrm{H} 4.29$, $\mathrm{Cl} 7.54, \mathrm{~N} 2.98$, O 13.62; found C 71.30, H 4.37, N 3.00.

7-Phenyl-benz[a]acridinium perchlorate $(\mathbf{1 5 g})$ in a yield of $82 \%$. M.p. $335{ }^{\circ} \mathrm{C}$. $-{ }^{1} \mathrm{H}$ NMR (400 MHz, $\left.\mathrm{CF}_{3} \mathrm{COOH}\right)$ : $\delta=7.37(\mathrm{~d}, J=12.8 \mathrm{~Hz}, 1 \mathrm{H}), 7.49(\mathrm{~d}, J=12.0 \mathrm{~Hz}, 1 \mathrm{H})$, $7.82-7.86(\mathrm{~m}, 2 \mathrm{H}), 8.02(\mathrm{~d}, J=9.6 \mathrm{~Hz}, 1 \mathrm{H}), 7.95-7.98(\mathrm{~m}$, $3 \mathrm{H}), 8.10-8.19(\mathrm{~m}, 2 \mathrm{H}), 8.25-8.31(\mathrm{~m}, 2 \mathrm{H}), 8.64(\mathrm{~d}, J=$ $12.8 \mathrm{~Hz}, 1 \mathrm{H}), 8.80(\mathrm{dd}, J=10.8 \mathrm{~Hz}, 1 \mathrm{H}) .-\mathrm{MS}((+) \mathrm{ESI})$ : $m / z=306.1$ (calcd. 306,38 for $\mathrm{C}_{23} \mathrm{H}_{16} \mathrm{~N}^{+},\left[\mathrm{M}-\mathrm{ClO}_{4}^{-}\right]^{+}$). $\mathrm{C}_{23} \mathrm{H}_{16} \mathrm{ClNO}_{4}$ (405.83): calcd. C 68.07, H 3.97, Cl 8.74, N 3.45, O 15.77; found C 68.01, H 3.97, N 3.52 . 
[1] G. Jones, S. P. Stanforth, Org. React., Vol. 49, John Wiley \& Sons, London, 1997, pp. 1 - 330.

[2] C. Grundmann, J.M. Dean, Angew. Chem. 1965, 77, 966-967; Angew. Chem., Int. Ed. Engl. 1965, 4, $955-$ 956.

[3] a) Y. Cheng, O. Meth-Cohn, D. Taylor, J. Chem. Soc., Perkin Trans. 1 1998, $1257-1262$; b) O. MethCohn, D. Taylor, J. Chem. Soc., Chem. Commun. 1995, 1463-1464; c) O. Meth-Cohn, Adv. Heterocycl. Chem. 1996, 65, 1-37; d) L. D. Patsenker, I. G. Ermolenko, I. A. Feyunyaeva, N. A. Popova, B. M. Krasovitskii, Chem. Heterocycl. Comp. 2000, 36, 623-625; e) L. D. Patsenker, I. G. Ermolenko, O. N. Lyubrenko, I. A. Fedyunyaeva, N. A. Popova, O.S. Galkina, A. V. Mazepa, B. M. Krasovitskii, Chem. Heterocycl. Comp. 2003, 39, 525-533; f) O. N. Semenova, Y. Y. Artyukhova, I. G. Yermolenko, L. D. Patsenker, Funct. Mater. 2003, 10, 730-738.

[4] a) J. Fabian, H. Nakazumi, M. Matsuoka, Chem. Rev. 1992, 92, 1197-1226; b) J. Fabian, J. Prakt. Chem. 1991, 333, 197-222.

[5] C. Bosshard, K. Sutter, P. Pretre, J. Hulliger, M. Flörsheimer, P. Kaatz, P. Günter, Adv. Nonlinear Optics, Vol. 1; Gordon \& Breach, Basel, 1995, pp. $1-$ 247.

[6] a) S. J. Chung, M. Rumi, V. Alain, S. Barlow, J.W. Perry, S. R. Marder, J. Am. Chem. Soc. 2005, 127, $10844-10845$; b) B. Strehmel, A. M. Sarker, H. Detert, ChemPhysChem 2003, 4, 249-259.

[7] H. Jenny, DE 1060375, 1961; Chem. Abstr. 1961, 55, 70758 .
[8] a) T. Mallegol, S. Gmouh, M. A. A. Meziane, M. Blanchard-Desce, O. Mongin, Synthesis 2005, 1771 - 1774; b) H.-Y. Wang, Austr. J. Chem. 2010, 63, $712-718$; c) H.-Y. Wang, Synth. Metals 2010, 160, 1065 -1072; d) Y.-J. Wang, H.-S. Sheu, C. K. Lai, Tetrahedron 2007, 63, 1695-1705; e) K. C. Majumdar, B. Chattopadhyay, P. K. Shyam, N. Pal, Tetrahedron Lett. 2009, 50, 6901-6905.

[9] R.S. Gairns, A. A. Watson, GB 2249789, 1992; Chem. Abstr. 1992, 117, 212314.

[10] T. B. Brantly, C. J. Fox, DE 1908346, 1969; Chem. Abstr. 1970, 72, 31416.

[11] a) K. C. Majumdar, B. Chattopadhyay, S. Chakravorty, N. Pal, R. K. Sinha, Tetrahedron Lett. 2008, 49, 7149 7152; b) H. Niegel, M. Zeiler, H. G. Wirth, DD 136146 , 1979; Chem. Abstr. 1979, 91, 212576.

[12] P. Gerstner, D. Rohde, H. Hartmann, Synthesis 2002 , $2487-2489$.

[13] U. Kuckländer, Acridine in Houben-Weyl, Meth. Organ. Chem., Hetarene II, Part 2, Vol. E7b (Ed. R. Kreher), Georg Thieme, Stuttgart, 1992, pp. 115-156.

[14] K. Lehmstedt, H. Hundertmark, Ber. Dtsch. Chem. Ges. 1931, 64, 2386-2394.

[15] I. Weeks, I. Beheshti, F. McCapra, A. K. Campbell, J. S. Woodhead, Clin. Chem. 1983, 29, 1474-1479.

[16] a) A. E. Porai-Koshiz, G. S. Ter-Sarkisyan, Isv. Akad. Nauk. SSSR, Ot. Khim. 1951, 771-776; b) A. E. PoraiKoshiz, G. S. Ter-Sarkisyan, Isv. Akad. Nauk. SSSR, Ot. Khim. 1951, 601-604; c) J. Griffith, Z. Li, Dyes Pigm. 1993, 21, $205-216$.

[17] C.-Y. Gao, X. Cao, L.-M. Yang. Org. Biomol. Chem. 2009, 7, $3922-3825$. 